\title{
An Empirical Study of Reliable Networking for Vehicular Networks Using IEEE 802.11n
}

\section{Seungbae Lee* and Alvin Lim}

Department of Computer Science and Software Engineering, 3101 Shelby Center for Engineering Technology,

Auburn University, Auburn, AL 36849-5347, USA

E-mail: \{sblee, limalvi\}@auburn.edu

*Corresponding author

\section{Qing Yang}

Department of Computer Science, 360 EPS Building, Montana State University, Bozeman, MT 59717, USA

E-mail: qing.yang@cs.montana.edu

\begin{abstract}
The IEEE 802.11n technology is becoming more and more prevalent in wireless networks due to its significant enhancements in network performance. To examine whether the reliability of $802.11 \mathrm{n}$ is sufficient for vehicular networks, we conducted extensive experiments on inter-vehicle and intra-vehicle communications in vehicular environments. From this empirical study, we found that 802.11n provides high performance with stable throughput and reliable coverage in most cases. However, 802.11n protocols do not detect frequent changes of propagation and polarization due to vehicle mobility and its rate adaptation algorithms improperly select multistream rates under channel fading conditions, although single-stream rates perform better. Moreover, an optimal antenna alignment that enables high throughput (HT) operation using parallel data streams needs further investigation in vehicular environments. Our findings have profound implications on the protocol design and appropriate configuration for reliable networking in vehicular networks using 802.11n.
\end{abstract}

Keywords: vehicular network; vehicular communication; performance; reliability; IEEE $802.11 \mathrm{n}$; MIMO; spatial stream; rate adaptation; MCS; antenna alignment.

Biographical notes: Seungbae Lee received the B.S. degree in computer science and statistics from Air Force Academy, South Korea in 1998 and the M.S. degree in technology management, economics and policy from Seoul National University, South Korea in 2008. Since 2010, he has been working towards the Ph.D. degree in computer science at Auburn University, USA. His current research interests include modeling and simulation of vehicular communications, and implementation of vehicular applications.

Copyright (c) 2012 Inderscience Enterprises Ltd. 
Alvin Lim is currently an associate professor of computer science and software engineering at Auburn University, USA. He received his Ph.D. degree in computer science from University of Wisconsin at Madison, USA in 1993. His research interests include self-organizing sensor networks, mobile and pervasive computing, network security, wireless networks, reliable and dynamically reconfigurable distributed systems, complex distributed systems, mobile and distributed databases, distributed operating systems, and performance measurement and analysis.

Qing Yang is currently a RightNow Technologies assistant professor of computer science at Montana State University, USA. He received his Ph.D. degree in computer science from Auburn University, USA in 2011. Before that, he obtained the B.E. and M.E. degrees in computer science and technology from Nankai University and Harbin Institute of Technology, China in 2003 and 2005, respectively. His research interests include wireless vehicular ad hoc networks (VANET), wireless sensor networks, network security and privacy, and distributed systems.

\section{Introduction}

The amount of information and services demanded from today's applications for intelligent transportation systems (ITS) in wireless vehicular networks has increased significantly over the past few years. These applications include data collection, data aggregation and translation, and information dissemination for autonomous driving, vehicle monitoring and diagnostics, and cooperative communications for operational instructions and safety information. All these applications require high performance, reliable networking and predictable coverage to realize their full potentials. Because they operate in a missioncritical mobile environment, information need to be instantly, constantly, and uninterruptedly delivered through wireless links between moving vehicles or between vehicles and the roadside infrastructure.

Currently, there are two distinct wireless technologies for vehicular communications: IEEE 802.11p (WAVE) and IEEE 802.11a/b/g/n (WiFi). $802.11 \mathrm{p} \mathrm{(2010)}$ is designed specifically for wireless access in vehicular environments, which uses the $5.9 \mathrm{GHz}$ band allocated for dedicated short range communication (DSRC). On the other hand, $802.11 \mathrm{a} / \mathrm{b} / \mathrm{g} / \mathrm{n}$ is originally developed for extending wireless communication links between indoor local area network (LAN) equipment. In this paper, we are interested in the network performance of 802.11n (instead of 802.11p) based vehicular communications for a couple of reasons. 802.11n (2009) has been widely used for wireless access in our daily life; the number of $802.11 \mathrm{n}$ users has become increasingly larger. As $802.11 \mathrm{p}$ standard was recently ratified and radios supporting the standard are expensive, it may be more practical and more cost-efficient to employ the prevalent $802.11 \mathrm{n}$ on users' vehicles. Because $802.11 \mathrm{n}$ can provide double or even higher performance with various diversity mechanisms for reliability, we believe $802.11 \mathrm{p}$ and $802.11 \mathrm{n}$ 
are complementary to each other. In effect, whether $802.11 \mathrm{p}$ or $802.11 \mathrm{n}$ is used depends on the type of application and effectiveness of deployment.

IEEE $802.11 \mathrm{n}$ is the latest standard of IEEE 802.11 (2007) technology, which promises high data rate, larger communication range and more reliable networking than previous $802.11 \mathrm{a} / \mathrm{b} / \mathrm{g}$ technologies. However, successful deployments of $802.11 \mathrm{n}$ in vehicular networks require an in-depth understanding of outdoor, especially vehicular, environments which may substantially affect network performance and reliability. For instance, it is difficult to maintain the same polarization between two communicating antennas to support reliable data communications. Moreover, the current rate adaptation algorithms used on $802.11 \mathrm{n}$ radios do not work properly in vehicular environments because they are designed for indoor use and based on previous $802.11 \mathrm{a} / \mathrm{b} / \mathrm{g}$ standards.

To investigate whether $802.11 \mathrm{n}$ technology can provide reliable networking for vehicular networks, we conduct numerous experiments and analyze the network performance of $802.11 \mathrm{n}$ vehicular communications. In this work, there are four major discoveries from our experiments which are the main contributions of this article.

- In most experiments of both inter-vehicle and intra-vehicle communications, IEEE $802.11 \mathrm{n}$ provides better performance showing stable throughput and reliable coverage. The performance is reliable at least between 0 to $30 \mathrm{Mbps}$ UDP bandwidth even if channel fading occurs.

- $802.11 \mathrm{n}$ wireless drivers and protocols do not detect polarization change or mismatch due to vehicle mobility on a hilly road or antenna misalignment and they do not have any algorithm or mechanism for detecting and handling this kind of complicated situation.

- To boost performance, the $802.11 \mathrm{n}$ rate adaptation algorithm frequently selects an improper dual data-stream rate of $802.11 \mathrm{n}$ in high UDP bandwidth (e.g, 40 - 100 Mbps) under channel fading conditions, in which dual data stream rates perform poorly and single-stream rates perform better.

- An optimal antenna alignment for successful 802.11n high throughput (HT) operation needs further investigation in vehicular environments. As typical IEEE $802.11 \mathrm{n}$ antennas only use either vertical or horizontal polarization with little multi-path signal reflection in outdoor environments, antenna alignment has a significant impact on creating separation between the antenna polarizations to support parallel data streams.

The rest of this paper is organized as follows. Section 2 introduces the critical techniques used in IEEE $802.11 \mathrm{n}$ for enhancing network performance. In Section 3, we present the distinct challenges of vehicular environments for $802.11 \mathrm{n}$ networking and discuss their effects on performance and reliability. Section 4 gives the details of our experiment setup including the hardware specification, network configuration and performance measurement procedure. Then, we analyze the reliability of $802.11 \mathrm{n}$ vehicular networks from our experimental results in Section 5. Related works are described in Section 6. Section 7 concludes our work with directions for future work. 


\section{Techniques for enhancing performance in IEEE 802.11n}

The success of the IEEE 802.11n standard is mainly due to its high throughput (up to $600 \mathrm{Mbps}$ ) from several key techniques for enhancing network performance, such as multiple-input multiple-output (MIMO), spatial multiplexing, advanced modulation and coding schemes (MCS), channel bonding and frame aggregation.

The IEEE $802.11 \mathrm{n}$ standard has a variety of enhancements including several techniques and mechanisms to achieve high throughput, which offers both benefits and challenges when it is applied to real wireless networks. This section introduces the key techniques in the $802.11 \mathrm{n}$ standard which are considered as the most important enhancements for improving network performance compared to the IEEE $802.11 \mathrm{a} / \mathrm{b} / \mathrm{g}$ standards.

\subsection{Multiple-input multiple-output (MIMO)}

The MIMO technology enables wireless devices to send two or more radio signals through multiple antennas and receive two or more radio signals coming from multiple antennas. MIMO is a significant innovation and a technology used by many non-IEEE 802.11 wireless networks, such as WiMax and LTE. Transmitting data through a single antenna can lead to a poor communication coverage area due to interferences, channel fading and multi-path errors. IEEE 802.11n uses multiple antenna polarities to transmit different versions of the same data. Thus, the receiver hears different strong signals in different parts of the coverage area and use the best available signals for communications. This results in more uniform coverage and higher throughput. In addition, 802.11n supports multiple spatial data streams that are simultaneously transmitted on the same channel through different antennas. The multiple data streams received from multiple receiving antennas are combined by advanced signal processing algorithms to reduce error probabilities and re-transmissions.

\subsection{Spatial multiplexing}

Spatial multiplexing with MIMO enables data to be split and transmitted via several independent data streams using spatial separation of polarizations from antennas, effectively increasing the throughput of a wireless channel. The spatial multiplexing functionality is available for most WiFi certified IEEE $802.11 \mathrm{n}$ products, except for some of handheld devices due to its limited number of antennas. Usually, it requires at least two spatial streams to be supported. Under most conditions, dual-stream spatial multiplexing can achieve double throughput compared to single-stream operation. However, the capability to send parallel data streams depends on the operating environment. In outdoor environments, it is difficult to create enough separation among the antenna signals to provide parallel data streams to support spatial multiplexing.

\subsection{Channel bonding and Frame aggregation}

IEEE 802.11n uses two different channel bandwidths: $20 \mathrm{MHz}$ and $40 \mathrm{MHz}$. By combining two adjacent $20 \mathrm{MHz}$ channels into a single $40 \mathrm{MHz}$ channel, $802.11 \mathrm{n}$ 
doubles the available data rate. Channel bonding is more effective in the $5 \mathrm{GHz}$ frequency since it allows 24 non-overlapping $40 \mathrm{MHz}$ channels, whereas the 2.4 $\mathrm{GHz}$ frequency has only 3 non-overlapping $20 \mathrm{MHz}$ channels. $802.11 \mathrm{n}$ also provides an option for combining multiple data frames ready for transmission into an aggregate frame. This reduces channel contention and delays by transmitting the aggregated frame in a single transmission on the channel.

\subsection{Modulation and coding scheme (MCS)}

IEEE $802.11 \mathrm{n}$ provides advanced modulation and coding schemes. MSC is a specification of the high-throughput (HT) physical layer (PHY) parameters that consists of number of data streams, modulation order (e.g., BPSK, QPSK, 16QAM, 64-QAM), forward error correction (FEC) coding rate (e.g., 1/2, 2/3, 3/4, $5 / 6$ ), and data rate. The $802.11 \mathrm{n}$ defines rate-dependent parameters for MCSs with indices 0 through 76 . For example, MCSs with indices 0 to 7 and 32 use single data stream and MCSs with indices 8 to 31 use multiple data streams with equal modulation. Table 1 lists MCS parameters for mandatory $20 \mathrm{MHz}$ bandwidth operation; MCS indices 0 to 15 are referred in our experiments. Therefore, rate adaptation algorithms for $802.11 \mathrm{n}$ work differently from previous protocols for $802.11 \mathrm{a} / \mathrm{b} / \mathrm{g}$, where the next best (higher or lower) rate is sequentially chosen. They need to select an MCS index among a wider range of combinations based on the current channel state, which means that a modulation type, a coding rate, the number of spatial streams, and a data rate are decided at the same time. Obviously, the MCS selection is directly linked to network performance.

Table 1 MCS parameters for mandatory $20 \mathrm{MHz}$

\begin{tabular}{c|ccccc}
\hline MCS index & Data streams & Modulation & Coding rate & \multicolumn{2}{c}{ Data rate (Mbps) } \\
\cline { 4 - 6 } & & & & 800 ns GI & $400 \mathrm{~ns}$ GI \\
\hline 0 & 1 & BPSK & $1 / 2$ & 6.5 & 7.2 \\
1 & 1 & QPSK & $1 / 2$ & 13.0 & 14.4 \\
2 & 1 & QPSK & $3 / 4$ & 19.5 & 21.7 \\
3 & 1 & 16-QAM & $1 / 2$ & 26.0 & 28.9 \\
4 & 1 & 16-QAM & $3 / 4$ & 39.0 & 43.3 \\
5 & 1 & 64-QAM & $2 / 3$ & 52.0 & 57.8 \\
6 & 1 & 64-QAM & $3 / 4$ & 58.5 & 65.0 \\
7 & 1 & 64-QAM & $5 / 6$ & 65.0 & 72.2 \\
8 & 2 & BPSK & $1 / 2$ & 13.0 & 14.4 \\
9 & 2 & QPSK & $1 / 2$ & 26.0 & 28.9 \\
10 & 2 & QPSK & $3 / 4$ & 39.0 & 43.3 \\
11 & 2 & 16-QAM & $1 / 2$ & 52.0 & 57.8 \\
12 & 2 & 16-QAM & $3 / 4$ & 78.0 & 86.7 \\
13 & 2 & 64-QAM & $2 / 3$ & 104.0 & 115.6 \\
14 & 2 & 64-QAM & $3 / 4$ & 117.0 & 130.0 \\
15 & 2 & 64-QAM & $5 / 6$ & 130.0 & 144.4 \\
\hline
\end{tabular}




\section{Challenges of IEEE 802.11n networking in vehicular environments}

The challenges of vehicular networking using IEEE 802.11n significantly differ from those of indoor networking. These differences arise from the following specific features of vehicular environments:

- In vehicular communications, the transmitter and receiver are mostly at the same height as the vehicles; for example, the height of most mid-size vehicles is 4 to $5 \mathrm{ft}$. Consequently, the propagation patterns are different from other mobile wireless links, where radios are placed high above the road level. The radio propagation of vehicular links is largely affected by horizontal obstacles at the same level and rising and falling slopes of hilly roads.

- The distance between the transmitter and receiver is strongly correlated to the speed of vehicles. A vehicle generally keeps a safe distance in proportion to its speed, which varies according to the traffic conditions: e.g., urban, suburban, rural, and highway environments. Due to variations on communication range, the channel quality frequently changes. Thus, communication performance is subject to wide fluctuations under the dynamic traffic condition.

- The $802.11 \mathrm{n}$ radios operate at high frequency, 2.4 or $5 \mathrm{GHz}$ band, which has high signal attenuation (or path loss) and few benefits of diffraction. Moreover, channel fading is faster than any other mobile communication because both the transmitter and receiver are simultaneously moving in most cases. Therefore, the maximal range for effective communications is much shorter and communication performance is usually degraded with a large variation over a short period of time.

- The propagation patterns in vehicular communications are significantly affected by vehicle geometry and antenna placement. A transmitter does not provide uniform coverage in a circular pattern, which actually creates coverage in intricate 3D patterns. Although rooftop mounting is intuitively considered as the best placement for vehicular antennas, there is still a need to empirically investigate the impact of vehicular antenna mounting on communication performance.

Specific capabilities and configurations of IEEE 802.11n for vehicular networks may differ from those of typical indoor networks. To exactly evaluate the performance and reliability of $802.11 \mathrm{n}$ in vehicular environments, we first need to understand these practical characteristics of vehicular networking.

\section{Experimental setup}

We conducted our experiments on a test track and a highway using two mid-size vehicles equipped with IEEE 802.11 -based radios. In this section, we describe in detail the setup of our experimental 802.11n vehicular network. 
The network performance of inter-vehicle communications was evaluated on the NCAT (National Center for Asphalt Technology) test track, which is an ovalshaped track in Alabama, USA, that is 1.7 miles $(2.7 \mathrm{~km})$ long. Since the track was designed as a comprehensive facility for various experimental purposes, it is a perfect place to run vehicular network tests; during our experiments, we could easily maintain both the speed of the vehicles and the distance between vehicles as intended. On the other hand, the performance of intra-vehicle communications was assessed on I-85, a major interstate highway in the southeastern USA, that accounts for vehicle mobility in real highway conditions.

Our experimental radio on the vehicle is a compact industrial platform (MS9A19) manufactured by MSI, which has an Intel Atom N270 1.6 GHz CPUembedded motherboard with a 2GB DDR2 memory and a compact flash (CF) 16GB storage. This radio has a mini PCIe interface for the wireless adapter and is powered by the vehicle battery. The operating system on the radio is a programmable Linux OS, Fedora 15 with Linux kernel 2.6.38, and supports the latest wireless driver sets. Using the radio, we could effectively configure and analyze experimental vehicular networks as planned.

To set up an IEEE 802.11n network on the experimental radio, a Atheros 802.11n chipset-based wireless adapter (Ubiquiti SR71-E) was used with the compat-wireless package, a set of Linux wireless code and drivers. The wireless adapter was chosen carefully to support dual-stream operation and access point (AP) configuration, because some of $802.11 \mathrm{n}$ radios do not support all the functionalities that the standard defines. Unlike other wireless drivers which simply extend the algorithms developed for the previous IEEE 802.11 standard, the compat-wireless provides better supports for new functions of the current 802.11 standard and its enhancements including 802.11n. For each wireless adapter, one of typical $7 \mathrm{dBi}$ fold-over dipole antennas with a magnetic mount (D-Link ANT24-0700) was selected, considering the vehicle mount. The antenna only uses either vertical or horizontal polarized omni-directional transmissions. Each radio is connected to two antennas placed 1 foot apart from the other.

When setting up the experiments, we observed that the performance of our radio may vary significantly. These variations are attributed to the channel condition and the network configuration. Although the wireless adapter supports the maximum data rate of $300 \mathrm{Mbps}$ at both $2.4 \mathrm{GHz}$ and $5 \mathrm{GHz}$ frequencies, we decided to set limits in order to obtain more reliable results with smaller variance. We used only $20 \mathrm{MHz}$ bandwidth with $800 \mathrm{~ns}$ guard interval (GI). Under the single or dual data-stream operation, the data rate is increased from 6.5 to $130 \mathrm{Mbps}$, which corresponds to the MCS indices from 0 to 15 . Also, we used only a 2.4 $\mathrm{GHz}$ frequency in our experiments, which provide better coverage with full data rates. Figure 1 shows the signal strength (measured in $\mathrm{dBm}$ ) at the receiver side in $2.4 \mathrm{GHz}$ (marked as a circle) and $5 \mathrm{GHz}$ (marked as a triangle) operations; this experiment was conducted on the test track. Our radio operating at $5 \mathrm{GHz}$ is significantly limited by distance, i.e., the maximum data-rate 130 Mbps (MCS 15) is only available when the distance is smaller than $100 \mathrm{ft}$.

To investigate IEEE $802.11 \mathrm{n}$ network reliability, Iperf, a standard network performance measurement tool, is employed to collect the result of network throughput and data loss. We developed scripts to control continuous Iperf test runs, which change the Iperf parameters every test run based on each 


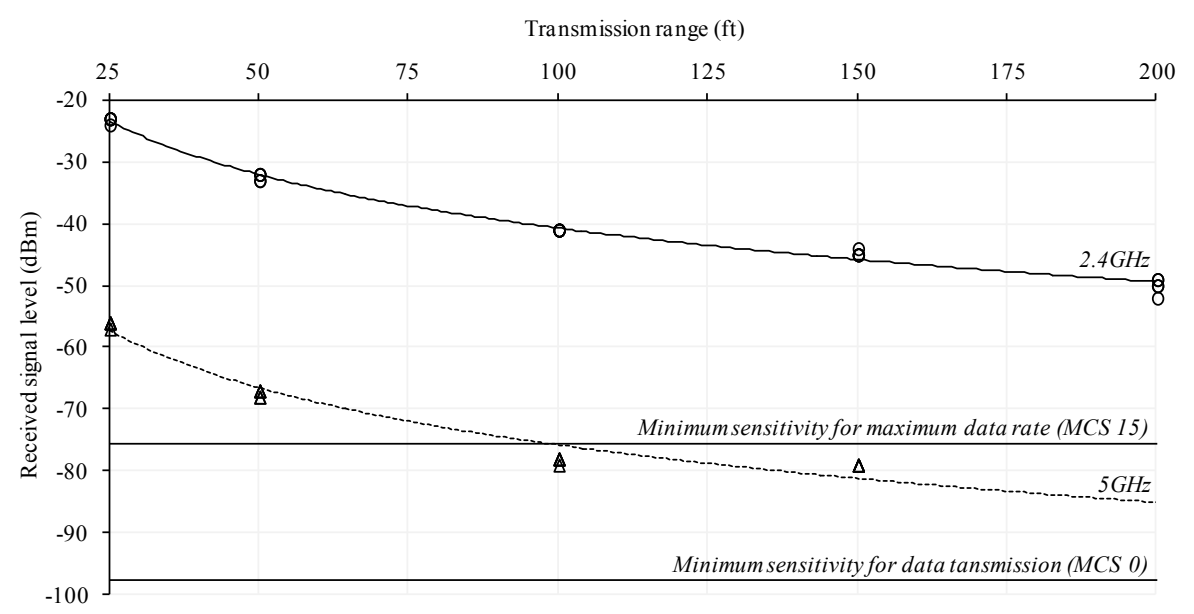

Figure 1 Signal strength reduction according to transmission range increase
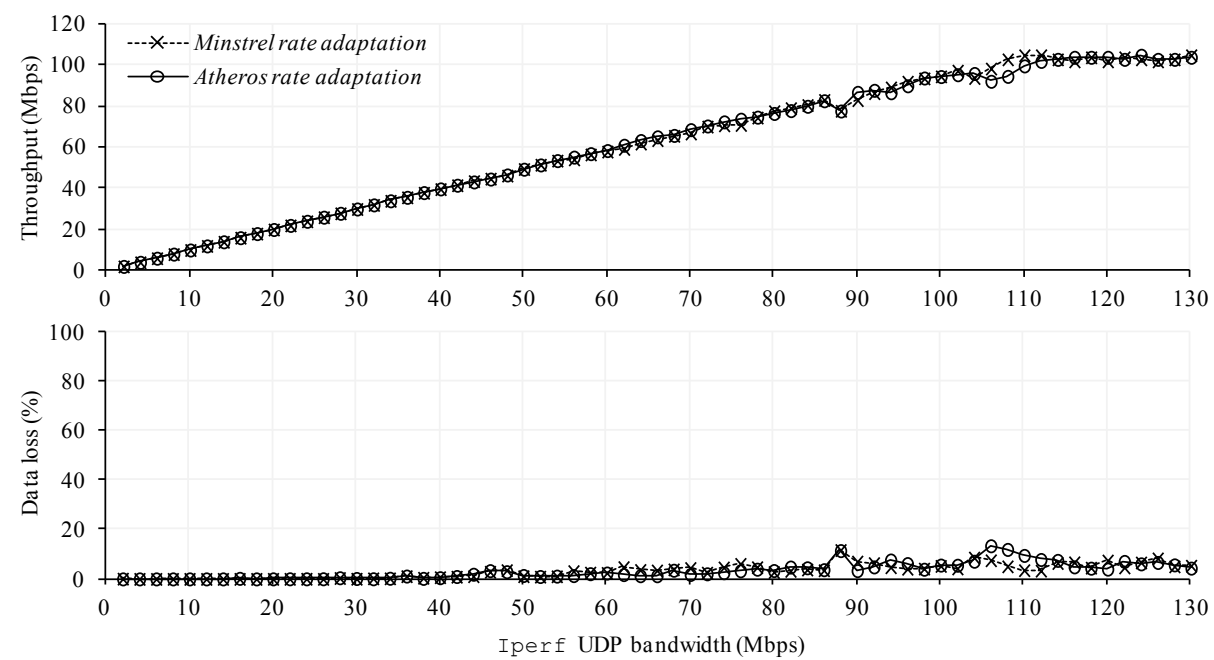

Figure 2 Typical network performance of experimental radios

experimental scenario. The script also collected wireless link statistics (e.g., data rate, received signal level, retransmission count, transmission failure count, etc.) from the Linux wireless driver and system messages from Linux kernel logs (e.g., hardware fault, wireless driver error, buffer overflow warning, etc.). In all our experiments, we did not observe any hardware fault, driver error, and buffer overflow message reported in the message logs. This indicates that there was no buffer overflow problem that could have caused data loss. 
We carried out the user datagram protocol (UDP) performance test using two radios in each experiment, because UDP is the most popular transport layer protocol in vehicular communications and the Iperf UDP test provides quality measurements suitable for evaluating network performance including throughput, loss and jitter. As the performance of a wireless link is usually affected by the surrounding channel conditions, the actual result provided by the Iperf UDP test may exhibit variations. To eliminate this effect, we first measured the network performance at $5 \mathrm{ft}$ range with an unobstructed line of sight (LOS) before each experiment, repeatedly running the Iperf tests and gradually increasing the UDPtest bandwidth every 30 seconds; we conducted our experiments only when all measurements and system messages were reported normally.

The typical network performance between two experimental radios is shown in Figure 2. One radio is configured as a transmitter (client) and the other as a receiver (access point) using Hostapd, a user-space Linux daemon for IEEE 802.11 access points and IEEE 802.1X/WPA/WPA2/EAP/RADIUS authentication servers. Linux wireless kernel supports two rate adaptation algorithms: Minstrel, commonly used for prevalent wireless adapters, and Atheros, specifically developed for Atheros chipset-based wireless adapters. Both protocols have similarities and differences, but they have not been completely optimized for the 802.11n High Throughput (HT) operation to date. Thus, there is no significant difference in the performance of $802.11 \mathrm{n}$ operation as shown in Figure 2. We decided to use the default rate adaptation algorithm on Linux wireless platforms, i.e., Minstrel, in all experiments. Moreover, we were able to observe that high-bandwidth UDP performance test involves data loss with variations but low-to-medium-bandwidth tests generate quite stable results. High-bandwidth transmission requires more complicated modulation and coding schemes and usually saturates the operating channel, which may cause data loss.

\section{Reliability analysis of IEEE 802.11n vehicular networks}

The use of IEEE 802.11n in vehicular environments has raised a number of questions such as how the specific configuration for 802.11n as applied to vehicular environments differs from the common indoor network configuration. This section examines the capabilities of $802.11 \mathrm{n}$ in vehicular environments focusing on network reliability. Section 5.1 and 5.2 introduce general performance results of our actual road experiments and two noteworthy cases of performance degradation in intervehicle and intra-vehicle communications. Section 5.3 investigates what causes the degradation in performance and how the reliability of $802.11 \mathrm{n}$ can be improved, through simulated experiments.

\subsection{Performance of inter-vehicle communications}

We performed our experiments on the test track using two mid-size vehicles equipped with one experimental radio, which has two antennas, under various scenarios to evaluate inter-vehicle communications in IEEE $802.11 \mathrm{n}$ vehicular networks. This section presents an interesting case among our inter-vehicle experiments. 

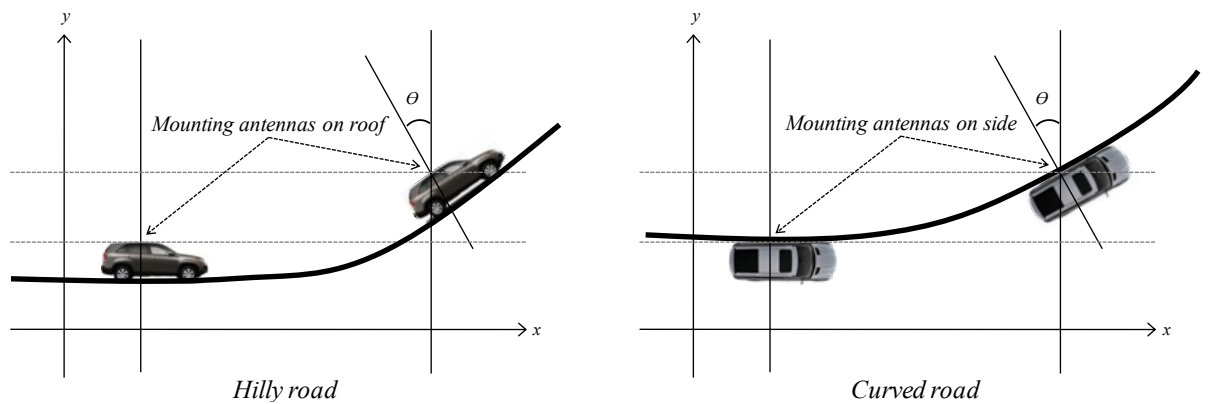

Figure 3 Antenna placement on experimental vehicles
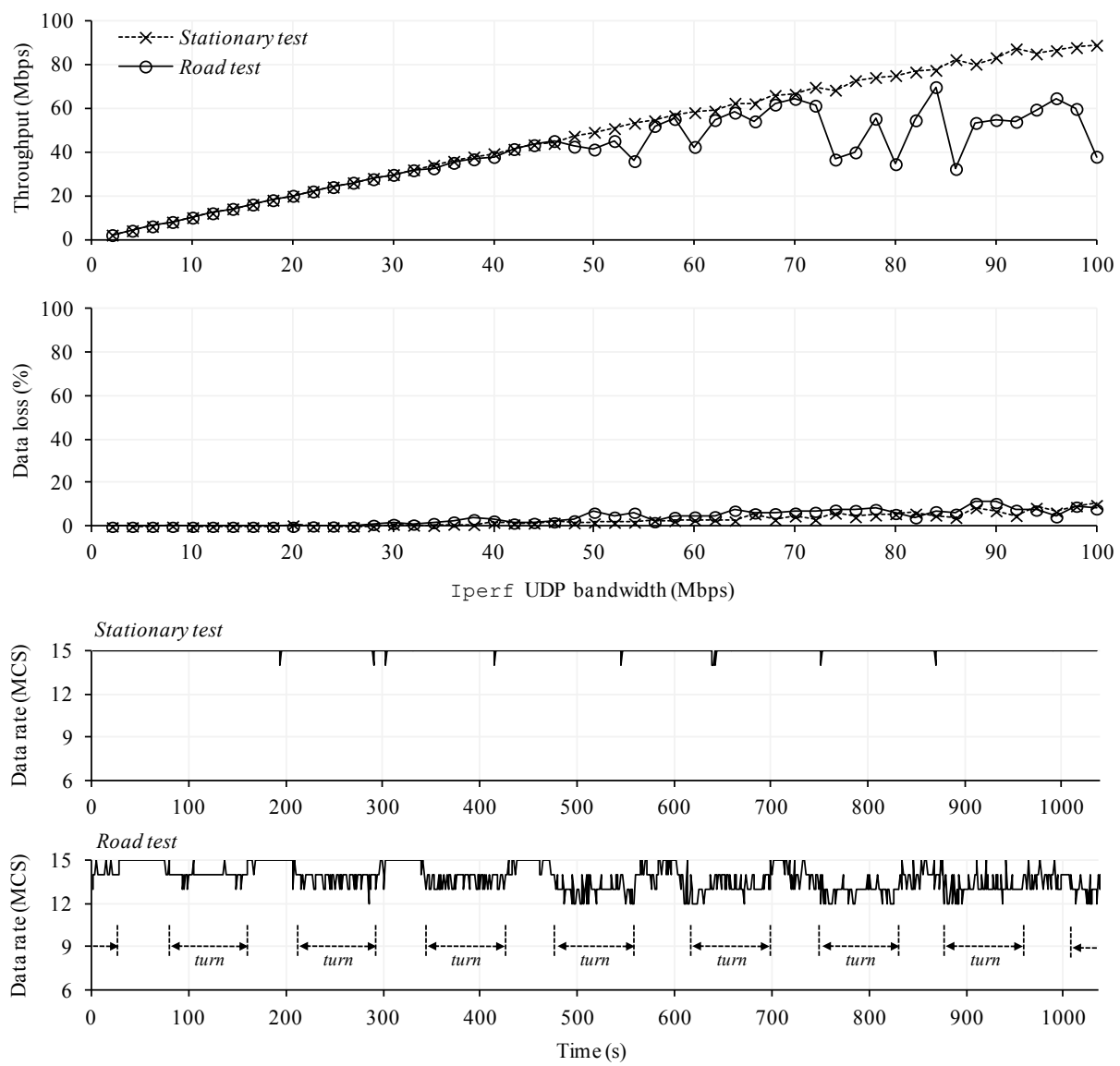

Figure 4 Network performance of inter-vehicle communications 
Most researchers have focused on two-dimensional vehicular environments. They often assumed that all the vehicles always move on level roads. However, actual vehicles may move on hilly roads with steep rising and falling slopes . To simulate a hilly road condition on our test tract, we placed two antennas on the side of each vehicle as illustrated by Figure 3. Since the oval-shaped 1.7-mile long test track consists of two straight and two curved sections, this antenna placement enables each lap to have two up-down hills. Two antennas were aligned parallel to the track.

In the road test, one vehicle went around the track continuously at $45 \mathrm{mph}$ and the other followed while keeping a $100 \mathrm{ft}$ distance from the leading vehicle. It was important to maintain the same distance between two vehicles so as to differentiate the hilly road effect from others such as path loss. The leader was configured as a receiver and the follower as a transmitter. We repeatedly ran the Iperf UDP tests, gradually increasing the UDP-test bandwidth every 10 seconds and observed the changes of throughput, datagram loss, and data rate (transmission rate). Before the road test, a stationary test was conducted on the open maintenance area of the test track to verify the network performance according to the configuration change.

Figure 4 indicates the changes in throughput, data loss, and data rate during the experiments. The stationary test gives stable throughput with low data loss as expected. Compared to the stationary test, the road test also provides good performance and reliability up to $45 \mathrm{Mbps}$ UDP test bandwidth, but we found performance variations in the mid-to-high bandwidth section of the road-test result. The data loss did not increase significantly in the section. We observed that only some of the datagrams corresponding to the UDP test bandwidth could be sent to the receiver for the duration of the test due to the reduced data rate and retransmission was successfully retried after unsuccessful transmission occurred during each Iperf test.

As no wireless error message including buffer overflow was observed in the message log, the data loss was attributed to the poor channel conditions. Another rationale that data loss is not due to buffer overflow is that the traffic load in the stationary test was the same as that in the mobile road test. Since the data loss in the stationary test was low, then there was no buffer overflow problem in both the stationary test and road test. We conclude that the high data loss in the road test beyond the Iperf test bandwidth of $50 \mathrm{Mbps}$ was due to poor channel conditions and not buffer overflow. Yet another rationale is that data rate controlled by Minstrel fluctuated whenever the vehicles turned around the curved track. This result suggests that the vehicle mobility on hilly roads substantially affects the performance and reliability of inter-vehicle communications in IEEE $802.11 \mathrm{n}$ networks which does not effectively adapt to this situation. We will continue to investigate the causes of this result in Section 5.3.

\subsection{Performance of intra-vehicle communications}

Vehicles contain a great number of electronic control units, sensors and actuators. The design, manufacture and installation of wiring harnesses for the transmission of power and data for all these components require considerable engineering efforts and costs. Replacing the number of wires in the harness with a wireless network 


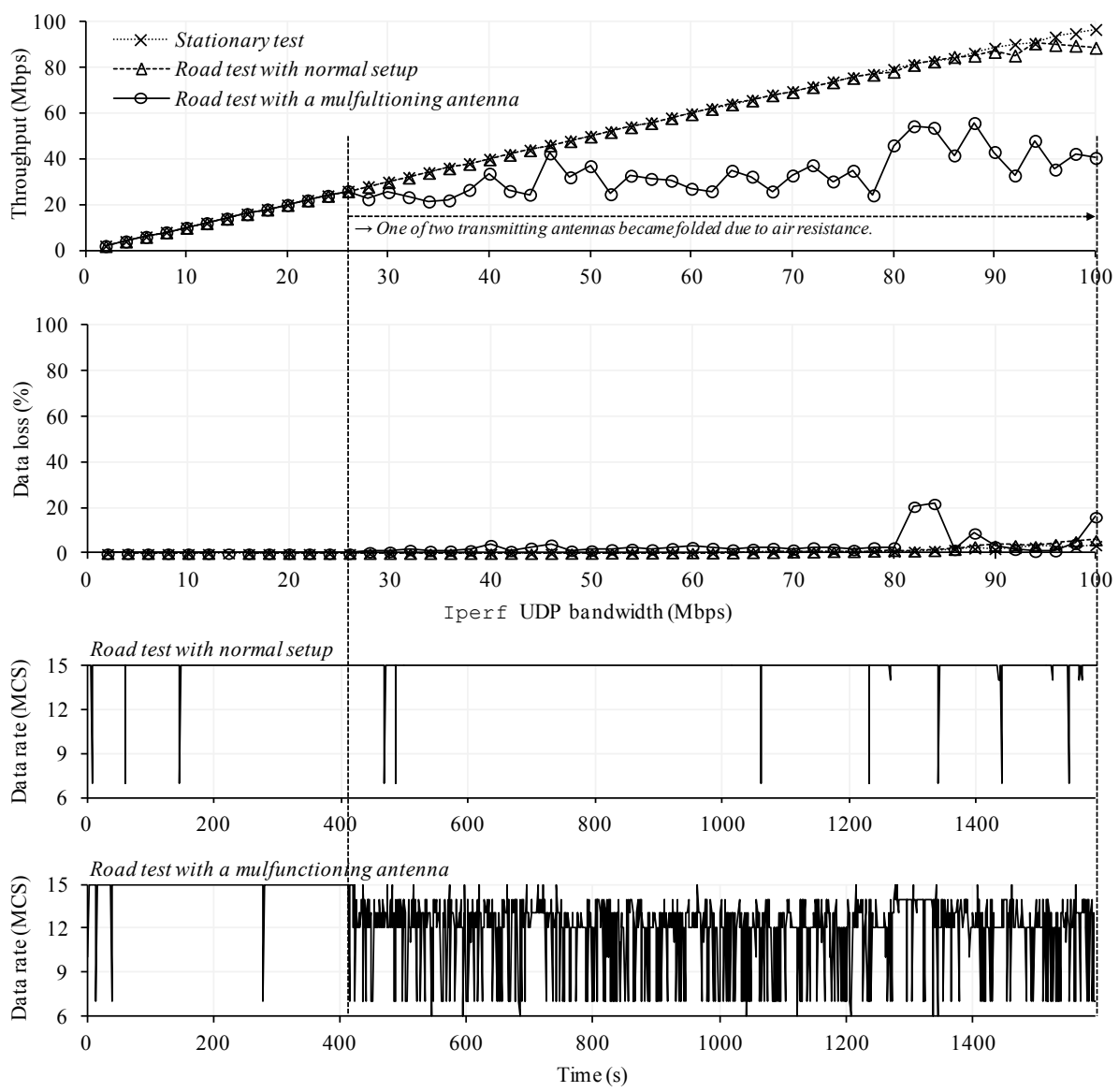

Figure 5 Network performance of intra-vehicle communications

can potentially provide part cost savings, assembly savings, and mass savings, which enables new technologies to be integrated into vehicles. We also carried out our experiments under numerous scenarios to access intra-vehicle communications in IEEE 802.11n configurations. This section describes a nontrivial case among our intra-vehicle experiments.

In the road tests, a mid-size vehicle equipped with two experimental radios moved at a speed up to $65 \mathrm{mph}$ on the highway. One radio was configured as a receiver with two antennas mounted on the front-side of the vehicle. The other was configured as a transmitter with two antennas placed on the rear side 5 $\mathrm{ft}$ apart from the receiver antennas. All the antennas were aligned parallel to the road. Since the antenna placement for intra-vehicle communications is largely constrained by the vehicle geometry, we experimentally mounted the antennas on the side of the vehicle to maximize the transmission range with unobstructed LOS. We repeatedly ran the Iperf UDP tests, gradually increasing the UDPtest bandwidth every 30 seconds and observed the performance change. Before 
the road tests, a stationary test was conducted in an open parking lot. In the majority of the cases, vehicle mobility did not influence the performance of intravehicle communications in the IEEE 802.11n configurations. Similar behaviors are observed in the stationary and road test as shown in Figure 5.

Figure 5 contains an interesting result from our intra-vehicle experiments. During the road test, one of two transmitting antennas became folded due to air resistance. Right after the unexpected mulfunction occurred, the throughput became unstable, more data loss was observed and the data rate oscillated between MCS index 15 and 7. The Minstrel rate adaptation algorithm of the transmitter had to decide on the number of data streams for data transmission: single data stream (i.e., MCS 15 to 8) or dual data stream (i.e., MCS 7 to 0). However, it could not make the correct decision, which resulted in the unstable performance. Obviously, it is preferable to limit the dual-stream operation to the single-stream in this particular situation with a malfunctioning antenna. This result indicates that IEEE 802.11n lacks the abilities to detect and cope with this kind of situation. We investigate the causes of this result in the next section.

\subsection{Effects of rate adaptation and antenna alignment}

It is generally accepted that wireless performance in vehicular networks is sensitive to radio propagation and polarization patterns. The use of multiple antennas to achieve spectral efficiency and diversity gains can provide robust connectivity for vehicular wireless links by alleviating the effects of fast fading due to frequent changes of propagation and polarization. Hence, IEEE $802.11 \mathrm{n}$ may be a better solution for vehicular wireless networks, which takes advantage of antenna diversity by employing MIMO technology to provide the performance and reliability required for efficient wireless links.

Contrary to our expectations, we identified a couple of problems in the deployment of IEEE 802.11n in vehicular networks based on our previous two cases. First, 802.11n protocols cannot detect polarization change or mismatch due to vehicle mobility on a hilly road or antenna misalignment. Second, the rate adaptation algorithm frequently selects an improper MCS index of 802.11n under the vehicular channel fading condition in mid-to-high bandwidth. Generally, these problems may be not important in indoor environments because $802.11 \mathrm{n}$ protocols are designed to use enough multi-path signal reflections from walls. This section analyzes the causes of the two previous cases and discusses how to maximize the benefits of $802.11 \mathrm{n}$ in vehicular networks through additional experiments.

In this section, all experiments were conducted on an open parking lot using two mid-size vehicles, each equipped with one radio and two antennas. The two vehicles are configured as the transmitter and receiver parked $50 \mathrm{ft}$ apart from each other. The antennas were mounted on the roof of each vehicle with unobstructed LOS between the transmitter and receiver. We simulated various conditions of polarization changes and mismatch by folding some of antennas and ran the Iperf UDP tests, gradually increasing the UDP-test bandwidth every 10 seconds to observe the performance of each configuration.

First, we aligned antennas so that a pair of antennas was not parallel to each other. Figure 6 shows three possible alignments and shows the results of experiments in Alignment \#1 configuration; similar results were observed in other 

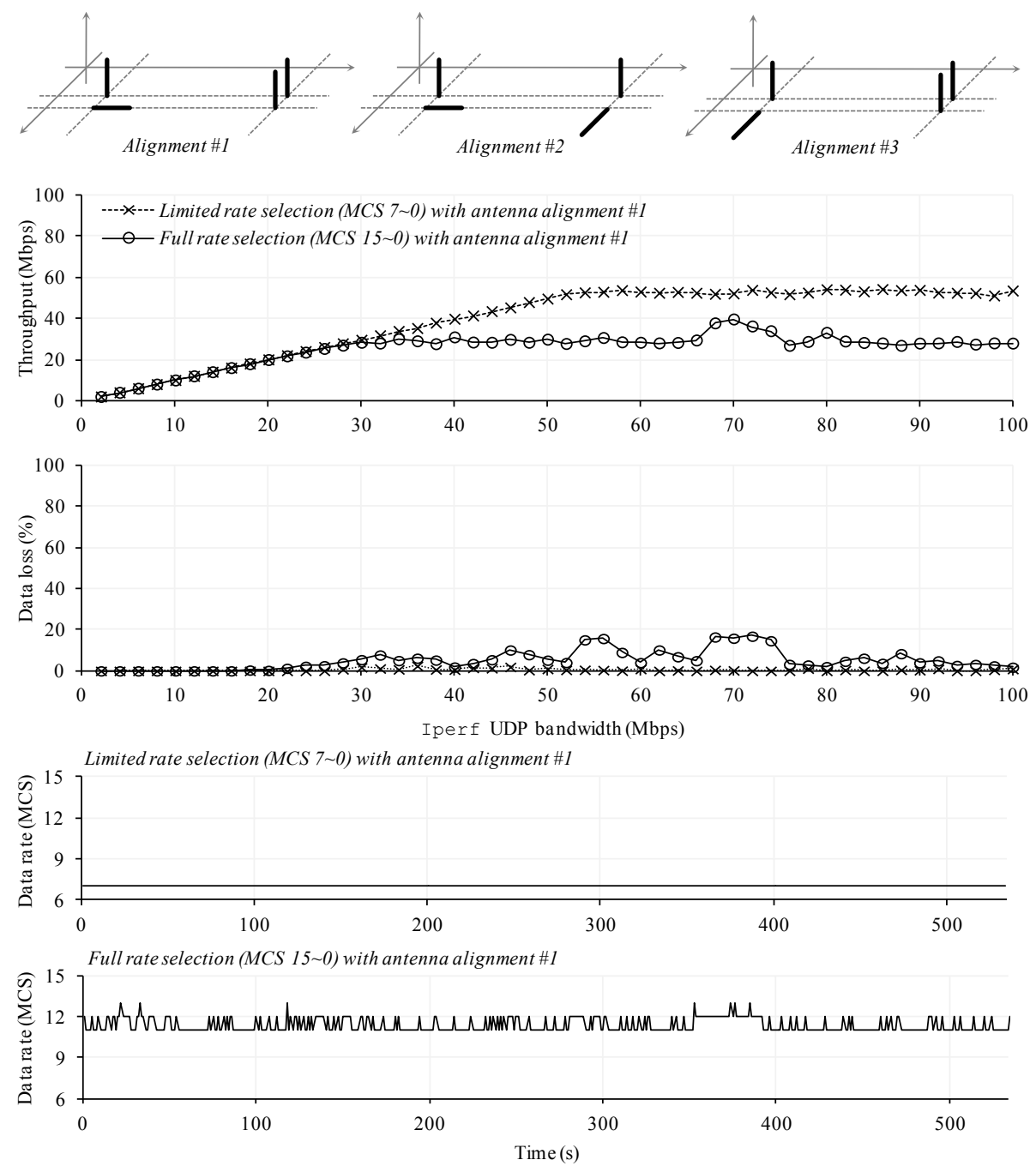

Figure 6 Performance comparison of rate-selection modes

experiments for Alignment \#2 and \#3 configurations, but those results are not presented for lack of space. We modified the Minstrel rate adaptation algorithm to control the number of data streams, i.e., the rate selection range could be limited. Minstrel selects MCS index 7 to 0 in the limited rate-selection mode, corresponding to single data-stream operation, or MCS index 15 to 0 in the full rate-selection mode, which uses single or dual data streams. The figure contains two results from different operating modes. A similar pattern as the two previous cases can be found in the result of the full rate-selection mode. Obviously, the rate adaptation algorithm with no limitation on the rate-selection range should have limited its rate to the single-stream scope in this configuration and provide one 

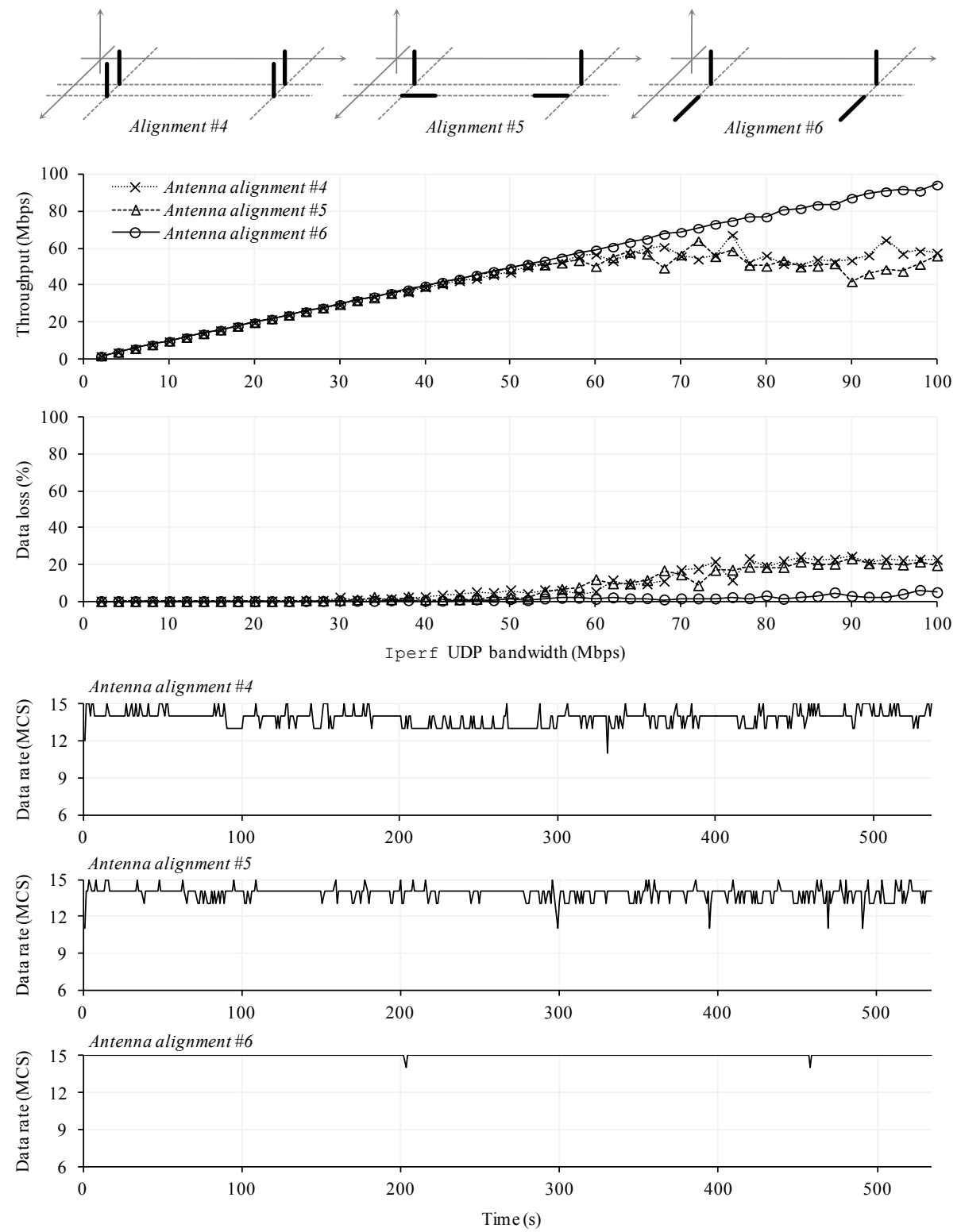

Figure 7 Performance comparison of antenna alignments

stronger direct spatial polarization. Instead, the rate adaptation algorithm simply reduced the data rate under dual steam operation by selecting the MCS index from 13 (104 Mbps) to 11 (52 Mbps), mostly MCS index 11, which causes performance degradation and data loss. On other hand, the rate adaptation algorithm with the limited rate-selection range achieved the maximum throughput by selecting the constant MCS index 7 (65 Mbps) in the same configuration. As evident from 
these experiments, the current rate adaptation algorithm needs to be enhanced in order to effectively cope with unexpected fading conditions caused by polarization change and mismatch under vehicle mobility.

Second, we conducted experiments to determine better alignments for vehicular antennas in IEEE 802.11n networks that will achieve the highest throughput. 802.11n supports spatial multiplexing that allows data to be spilt and transmitted via two independent data streams using spatial separation of polarizations from two pair of antennas. We aligned antennas so that two pairs of parallel antennas were placed. Figure 7 illustrates three possible alignments and displays the results of experiments in the three configurations. Alignment \#4 is generally considered a basic setup for $802.11 \mathrm{n}$ antennas, but actually provides no better performance and reliability in vehicular environments compared to other alignments. Empirically, we found that Alignment \#6 is the optimal alignment for vehicular 802.11n networks. As typical 802.11n antennas, including our experimental antennas, only use either vertical or horizontal polarization based on its alignment with little multi-path signal reflection, antenna alignment has a significant impact on the performance of multiple data-stream operation in vehicular environments.

Through extensive experiments with realistic scenarios, we evaluated the performance of IEEE 802.11n in vehicular environments focusing on reliable networking. In most cases, 802.11n provides better performance showing higher throughput with reliable coverage, especially between 0 to $30 \mathrm{Mbps}$ UDP bandwidth. However, we identified the problems of current $802.11 \mathrm{n}$ rate adaptation algorithms and the effects of vehicular antenna alignment. To successfully deploy $802.11 \mathrm{n}$ and effectively reap its benefits in vehicular networks, an innovative rate adaptation algorithm for vehicular environments needs to be developed that will adapt to channel conditions under vehicle mobility and vehicular antenna installation has to be treated with great care based on better understanding of vehicular environments.

\section{Related work}

The general overview of IEEE 802.11n development is completely discussed by Perahia (2008), in which 802.11 enhancements for high throughput and robustness of the wireless link are described. From this article, we found the key techniques of $802.11 \mathrm{n}$, including MIMO, spatial multiplexing and advanced MCSs, can support vehicular networks by providing better performance and reliability. The current $802.11 \mathrm{n}$ systems, however, have not comprehensively supported all functionalities of the standard. Pefkianakis et al. (2010) pointed out that existing rate adaptation algorithms do not consider the characteristics of diversity-oriented single-stream operation and spatial multiplexing driven dual-stream operation in MIMO systems, and thus suffer from severe performance degradation. To address this problem, Halperin et al. (2010) developed a packet delivery prediction algorithm using standard Channel State Information (CSI) measurements that 802.11 n radios provide, which can be applied to many classic problems including rate adaptation, transmit power tuning, and channel and antenna selection in 802.11n communications. Although there have been several research efforts to design specialized protocols for vehicular environments based on the IEEE 802.11 
standard as summarized by Jarupan and Ekici (2011), no work has concentrated on the key enhancements of 802.11n to implement reliable networking for vehicular networks.

As Molisch et al. (2009) and Mecklenbrauker et al. (2011) investigate in their paper, the characteristics of vehicular channels, such as temporal variability and propagation pattern, differ from other mobile communication channels, e.g., cellular communications, and vary in the properties of operating environments, e.g., highways, urban streets. Otto et al. (2009) measured how actual IEEE 802.11b propagation varies in different environments, especially between line-of-sight and non-line-of-sight communication. To address this issue, several experimental studies have been conducted using their own radios or $802.11 \mathrm{a} / \mathrm{b} / \mathrm{g}$ radios, which focus on rate adaptation and antenna placement. Camp and Knightly (2008) found that SNR-based rate adaptation algorithms, compared to loss-based protocols, are better able to track mobility and have higher robustness and accuracy in the presence of fast fading and interference. Eggers et al. (2007) placed antennas inside the vehicle and on the rooftop in their experiments, and concluded that the inside antennas lead to higher path loss and stronger multipath propagation. Kaul et al. (2007) found the antenna propagation pattern and packet error rate in vehicular communications are very sensitive to the antenna position. However, it is rare to find a related study regarding rate adaptation and antenna alignment for $802.11 \mathrm{n}$ MIMO and spatial streams in vehicular environments, even in outdoor conditions.

In summary, most prior works on applying IEEE 802.11 technology to vehicular networks focus on outdated 802.11 standards while optimal configurations for $802.11 \mathrm{n}$ in vehicular environments have not been explored. To the best of our knowledge, we are the fist to empirically investigate the network reliability of intervehicle and intra-vehicle communications in $802.11 \mathrm{n}$ based vehicular networks.

\section{Conclusion}

From thorough empirical studies, the benefits and limitations of applying IEEE 802.11n technology to vehicular networks were identified. To accomplish the full network capacities provided by $802.11 \mathrm{n}$, a couple of issues, such as rate adaptation and antenna alignment, need to be addressed for reliable networking in vehicular environments. $802.11 \mathrm{n}$ provides not only a variety of technical enhancements that ensure reliable network performance but also better operational efficiencies compared to other wireless technologies. However, successful deployments of $802.11 \mathrm{n}$ in vehicular networks require an in-depth understanding of the vehicular wireless communication environments and the advanced techniques used in 802.11n for enhancing network performance. In our future work, a sophisticated rate adaptation algorithm with an optimal antenna design will be explored to cope with channel fading due to vehicle mobility. Since $802.11 \mathrm{n}$ technology is becoming more prevalent and is in a constant state of improvement, future vehicular networks have to consider connection with existing $802.11 \mathrm{n}$ infrastructures and $802.11 \mathrm{p}$ should be enhanced by employing advanced techniques of $802.11 \mathrm{n}$ to achieve better performance and reliability. 


\section{Acknowledgements}

This research is supported in part by the National Transportation Research Center, Inc. under contract NTRCI-DTRT-06-G-0043-04-U29 and NTRCI-DTRT06-G-0043-04-U31 under a grant from the U.S. Department of Transportation Research and Innovative Technology Administration/FHWA (\#DTRT-06-G-004303-U24-01-01).

\section{References}

(2007). IEEE Standard for Information technology - Telecommunications and information exchange between systems - Local and metropolitan area networks Specific requirements - Part 11: Wireless LAN Medium Access Control (MAC) and Physical Layer (PHY) Specifications. IEEE Std 802.11-2007 (Revision of IEEE Std 802.11-1999), pages $1-1184$.

(2009). IEEE Standard for Information technology - Telecommunications and information exchange between systems - Local and metropolitan area networks Specific requirements - Part 11: Wireless LAN Medium Access Control (MAC) and Physical Layer (PHY) Specifications - Amendment 5: Enhancements for Higher Throughput. IEEE Std 802.11n-2009 (Amendment to IEEE Std 802.11-2007 as amended by IEEE Std 802.11k-2008, IEEE Std 802.11r-2008, IEEE Std 802.11y2008, and IEEE Std 802.11w-2009), pages $1-502$.

(2010). IEEE Standard for Information technology - Telecommunications and information exchange between systems - Local and metropolitan area networks Specific requirements - Part 11: Wireless LAN Medium Access Control (MAC) and Physical Layer (PHY) Specifications - Amendment 6: Wireless Access in Vehicular Environments. IEEE Std 802.11p-2010 (Amendment to IEEE Std 802.11-2007 as amended by IEEE Std 802.11k-2008, IEEE Std 802.11r-2008, IEEE Std 802.11y2008, IEEE Std 802.11n-2009, and IEEE Std 802.11w-2009), pages 1 -51.

Camp, J. and Knightly, E. (2008). Modulation Rate Adaptation in Urban and Vehicular Environments: Cross-layer Implementation and Experimental Evaluation. In 14th ACM international conference on Mobile computing and networking, MobiCom '08, pages 315-326.

Eggers, P., Brown, T., Olesen, K., and Pedersen, G. (2007). Assessment of Capacity Support and Scattering in Experimental High Speed Vehicle to Vehicle MIMO Links. In Vehicular Technology Conference, 200\%. VTC2007-Spring. IEEE 65th, pages $466-470$

Halperin, D., Hu, W., Sheth, A., and Wetherall, D. (2010). Predictable 802.11 Packet Delivery from Wireless Channel Measurements. SIGCOMM Comput. Commun. Rev., 40(4):159-170.

Jarupan, B. and Ekici, E. (2011). A survey of cross-layer design for VANETs. Ad Hoc Networks, 9(5):966-983.

Kaul, S., Ramachandran, K., Shankar, P., Oh, S., Gruteser, M., Seskar, I., and Nadeem, T. (2007). Effect of Antenna Placement and Diversity on Vehicular Network Communications. In Sensor, Mesh and Ad Hoc Communications and Networks, 2007. SECON '07. 4th Annual IEEE Communications Society Conference on, pages $112-121$. 
Mecklenbrauker, C., Molisch, A., Karedal, J., Tufvesson, F., Paier, A., Bernado, L., Zemen, T., Klemp, O., and Czink, N. (2011). Vehicular channel characterization and its implications for wireless system design and performance. Proceedings of the IEEE, 99(7):1189 -1212.

Molisch, A., Tufvesson, F., Karedal, J., and Mecklenbrauker, C. (2009). A Survey on Vehicle-To-Vehicle Propagation Channels. Wireless Communications, IEEE, 16(6):12-22.

Otto, J., Bustamante, F., and Berry, R. (2009). Down the Block and Around the Corner The Impact of Radio Propagation on Inter-vehicle Wireless Communication. In Distributed Computing Systems, 2009. ICDCS '09. 29th IEEE International Conference on, pages 605-614.

Pefkianakis, I., Hu, Y., Wong, S., Yang, H., and Lu, S. (2010). MIMO Rate Adaptation in 802.11n Wireless Networks. In 16th annual international conference on Mobile computing and networking, 2010. MobiCom '10, pages 257-268.

Perahia, E. (2008). IEEE 802.11n Development: History, Process, and Technology. Communications Magazine, IEEE, 46(7):48-55. 